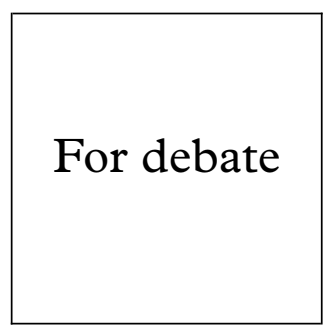

\title{
Should preventive antiretroviral treatment be offered following sexual exposure to HIV?
}

\author{
Not yet!
}

\author{
Barry Evans, Janet Darbyshire, Jonathan Cartledge
}

\begin{abstract}
Background
Current transmission of HIV within the United Kingdom, especially among men who have sex with men, is unacceptably high. ${ }^{1}$ Every potential way of reducing transmission should be explored and, if likely to be effective, introduced as rapidly as possible. However, we must be reasonably sure of the benefits and risks of any new intervention such as post-exposure prophylaxis (PEP). If there were no side effects from the drugs, if the logistical problems of getting treatment soon enough after exposure could be overcome, if it could be shown that no increase in behavioural risks occurred as a result, if there was no risk of an increase in resistance to the drugs in the population, and if, in these money oriented times, the costs were low we could proceed in spite of a lack of convincing evidence about the efficacy of the intervention. Unfortunately, none of these criteria are fulfilled for PEP for sexual exposure; indeed, there are no data at all on efficacy. If, in these circumstances, PEP for sexual exposure is implemented half heartedly this will be the worst possible way of proceeding.
\end{abstract}

\section{Occupational exposure: interpretation of the case-control study}

In any discussion on the efficacy of PEP, the data on occupational exposure are cited. The single case-control study on which all of the evidence is based has just been published in full ${ }^{2}$ after its initial publication in the Morbidity and Mortality Weekly Report in 1995. ${ }^{3}$ Everyone acknowledges that the study has design faults, especially that cases and controls were drawn from different populations! Further, the different methods of reporting - active for cases but voluntary for controls - is likely to have led to biases. For example, in the control group those individuals who were treated would be more likely to be remembered and therefore reported. The authors tried to identify biases in relation to the use of zidovudine between cases and controls which could account for the differences, but were not able to- this does not mean that they do not exist. However, in $70 \%$ of cases and controls the source patient was taking zidovudine at the time the healthcare worker was exposed and for these there was a high probability that the virus transmitted was resistant to the drug. The authors conclude that the efficacy may, therefore, be even higher in preventing transmission of sensitive virus. However, it is difficult to believe that the use of zidovudine led to $80 \%$ efficacy if resistance levels approached $70 \%$ and, therefore, an alternative interpretation is that the differences were, in fact, more likely to be due to the biases inherent in the study design even if the authors could not identify these. A randomised controlled trial, which would have provided an unbiased answer, was unfortunately abandoned because of the failure to recruit. Whatever the difficulties in interpretation, the case-control study provides the only data on which to base decisions and the weight of scientific opinion is to recommend prophylaxis after occupational exposure as the potential benefits in this population are likely to outweigh the risks. ${ }^{4}$ At this stage, a randomised placebo controlled trial would be unlikely to be ethically acceptable in the occupational setting.

\section{Extrapolating from occupational to sexual exposure}

In considering the case for PEP for sexual exposure, the many differences between the two types of exposure and our knowledge about them must be reviewed. The basis for caution over the use of PEP for sexual exposure stems from doubts about the interpretation of the casecontrol study and from its applicability to non-occupational exposure. Its introduction on the far larger scale potentially required for sexual exposure without attempting a randomised trial, in spite of all the difficulties, is hard to justify. The implications of a policy of PEP for healthcare workers, which is likely to affect, at most, a couple of hundred people in the United Kingdom each year, are not too great. However, the consequences of introducing PEP for potentially tens of thousands who are at risk from sexual exposure, or think they may have been, are enormous. Since the early 1980 s four healthcare workers have become HIV positive after occupational exposure in the United Kingdom ${ }^{5}$ compared with about 25000 who have been infected through sexual exposure. ${ }^{6}$ These figures give some idea of the relative order of magnitude of demand for PEP, but also of its potential to make a contribution to limiting sexual transmission - if it was demonstrated to be effective and more importantly was taken by the right people at the right time. However, the use of condoms is likely to be just as, if not more, effective and much cheaper and simpler! 


\section{Risks of infection following sexual exposure}

The highest risk of sexual transmission of HIV is from unprotected receptive anal intercourse where the insertive partner is infected with HIV with a lower, but still significant, risk when the receptive partner is infected. Lower risks of transmission have been reported for couples engaging in unprotected vaginal intercourse when the male partner is infected and lower again when the female is infected. Realistic estimates of the levels of risk are notoriously difficult to obtain and the results of studies difficult to interpret. The risks are dependent on a variety of other factors including the presence of other sexually transmitted infections, especially those associated with ulceration, and the viral load in the plasma, genital secretions, or semen of the source case. It is difficult therefore to estimate the transmission risk per sex act. However, if the estimates recently summarised ${ }^{7}$ are of the correct order of magnitude then for unprotected vaginal intercourse in the United Kingdom, where the chance of ulcerative STDs is low, several hundred courses of PEP would be taken needlessly to prevent one seroconversion (transmission risk between 0.01 and 0.001). The risks in the seroconversion phase of HIV or when other STDs act as cofactors would change this estimate but, conversely, if the infective source had a low viral load then the risks might be even lower.

If PEP were used in exposures where the HIV status of the partner was unknown then the estimates are orders of magnitude lower. In most communities in the United Kingdom, where HIV seroprevalence in the heterosexual population is low, the risk from a partner of unknown HIV infection status may be of the order of 100 times lower and outside major urban centres lower still. In this scenario tens of thousands of courses of PEP would be given to prevent one transmission. Even if cost considerations are not taken into account the side effects of such courses of treatment must outweigh the potential benefits of PEP for such sexual exposures. Risks will depend on the background prevalence of HIV in the population and knowledge of this will be crucial if PEP is considered without knowledge of the HIV status of the partner. It will seldom, if ever, be justified in heterosexual exposures where the HIV infection status of the partner is unknown.

Calculations for homosexual men engaging in unprotected anal sex in London or other major cities give different estimates but the risk of transmission must always be considered in relation to the risk of taking potentially toxic drugs for several weeks. A risk per sex act of about 0.01 for receptive anal intercourse would result in an average of 100 courses of treatment per case prevented assuming treatment could be obtained promptly and was completely effective. With partners of unknown status, even in highest prevalence areas with HIV rates of around $10 \%$ in homosexual men attending STD clinics, this would result in an average of 1000 courses of treatment per case prevented. Elsewhere the potential benefits would be yet lower. It would be difficult to justify the use of potentially toxic drugs for any exposure involving a sexual partner of unknown HIV status when the benefit-risk ratios are so low.

Without doubt routine condom use would be more effective than any PEP, and it has no side effects! In the current state of knowledge it would be wise to be extremely cautious about the widespread introduction of PEP even following relatively high risk sexual exposure. If a healthcare worker is exposed in an occupational setting, the source patient can be counselled and tested, if their HIV status is not already known, and if they refuse testing, a risk assessment can be carried out. If the HIV status of a sexual partner is unknown-and counselling and testing seem unlikely to be an option in most circumstances-or "known" only from hearsay, an enormous number of drugs may be used in low or no risk situations.

\section{Logistic problems}

Perhaps the greatest practical obstacle to the introduction of PEP is the logistics of counselling the individual and starting PEP within a few hours - not that we have any idea what is the maximum time between exposure and PEP when it is likely to be of benefit. The relatively limited animal data, which provide little support for the concept of PEP, suggest that antiretroviral drugs need to be given very soon after exposure to have any impact. If we follow this path, 24 hour access to therapy would be required and the costs of this will considerably increase the total costs of its widespread introduction. If we are convinced about the efficacy of PEP, these problems must be overcome. Until we are, the expense is hard to justify, the impetus to overcome the logistics is small, and there is likely to be a half hearted approach to implementation. ${ }^{8}$

\section{Potential risks of PEP}

One of the concerns about the widespread introduction of PEP is the risk that it may lead to the development of HIV strains resistant to one or more of the relatively small number of drugs currently available for the treatment of HIV infection. This would be unlikely to occur if a short course of effective therapy was taken - for example, for 4-6 weeks-a duration based on little evidence. However, there are a number of factors which may increase the risk of resistance. Firstly, we know from experience with occupational exposure that many healthcare workers do not take the full course for various reasons. Major or, more likely, minor side effects may occur and cause the discontinuation of one or more drugs. The source case is likely to have had therapy with several drugs and may, therefore, have transmitted resistant strains so that the therapy may be less effective than anticipated and resistance may be more likely to occur. If individuals have frequent sexual exposures, as many do, they may present for recurrent courses of therapy and this may increase the risk of the development of resistance. 


\section{The need for a randomised controlled trial}

The use of PEP would be justified if we knew it worked-only a randomised trial would determine this reliably. It might also provide some guidance on who to offer it to. With determination, the difficulties experienced in recruiting for the occupational trial could be overcome as the numbers of individuals exposed must be much greater. A pilot study should be set up soon and an international randomised placebo controlled trial could answer the question in a rapid and timely manner. Serodiscordant couples could for instance be recruited and provided with placebo/PEP packs for use after condom breakage. Counselling would be essential about the unknown efficacy and the need to continue safer sex. People presenting after a potential risky exposure could be recruited. Ideally, the HIV status of the partner should be determined in as high a proportion as possible and the presence of sexually transmitted diseases in both partners as this will influence transmission. If PEP is given "on demand" the chances of conducting trials on which policy should be based will be jeopardised. There is a risk that if clinicians begin to prescribe PEP and individuals do not seroconvert, faith in the "efficacy" of the therapy may become established even though almost all individuals would not have seroconverted even without PEP. However, if the situation is explained and the current uncertainty about the potential risks and benefits communicated to the potential participants, a significant number may be willing to take part in a randomised trial. There is no doubt that much time is required to discuss with individuals the need to weigh up a very small risk that may be reduced by an unproved treatment against the, again unknown, potential long term toxicity of that treatment so that they are in a position to make an informed decision. Such a decision is difficult enough for a clinician let alone a distressed patient. The availability of a randomised trial relieves both the clinician and the individual from the responsibility of making such a decision. There is no doubt about the urgency of conducting such a trial. We will have only one chance to undertake it - if we fail and, on a widespread basis, introduce an expensive potentially toxic therapy in the absence of evidence of efficacy, we will not only fail our patients now, but also in the future. Far from being unethical to withhold PEP after sexual exposure, it would be unethical to introduce it in response to demand from patients. However, it would be equally wrong not to actively encourage it if we were sure of its efficacy. Caution is justified and the questions raised by patient groups and clinicians remain which only a randomised controlled trial will answer reliably. If we do not move quickly to attempt such a trial we may do more harm than good.

\section{The need for surveillance}

While discussions are ongoing about the way forward to set up a trial, surveillance of the size of the problem in the United Kingdom should be undertaken as rapidly as possible. If a trial proves to be impossible to organise - and we shouldn't give up too easily-then surveillance will be the only way to obtain data which may help in clinical decision making. An initial survey is currently being conducted via the British Cooperative Clinical Group (a subgroup of the MSSVD). The results of this survey will determine what current practice and demands are and will aid further plans.

1 Macdonald ND. AIDS and HIV infection acquired through sexual intercourse between men. Commun Dis Rep CDR Rev 1997;7:R123-4.

2 Cardo DM, Culver DH, Ciesielski CA, et al. A case control study of HIV seroconversion in healthcare workers after percutaneous exposure. N Engl f Med 1997;337:1485-90.

3 Centers for Disease Control. Case control study of HIV seroconversion in HCW/s after percutaneous exposure to HIV infected blood-France, United Kingdom and United States, January 1988-August 1994. MMWR 1995;45:92933.

4 UK Health Departments. Guidelines on post exposure prophylaxis for HCWs occupationally exposed to HIV. prophylaxis for HCWs occupationally exposed to

5 Heptonstall J, Gill ON, Porter K, et al. HCWs and HIV: surveillance of occupationally acquired HIV in the UK. Commun Dis Rep CDR Rev 1993;3:R147-53.

6 Allardice G, Hughes G. AIDS and severe HIV disease; combined predictions for the UK to 1999. Commun Dis Rep CDR Rev 1996;6:R192-4

7 Mastro TD, de Vincenzi I. Probabilities of sexual HIV-1 transmission. AIDS 1996;10(suppl A):575-82.

8 Pinkerton SD, Holtgrave DR, Pinkerton HJ. Cost effectiveness of chemoprophylaxis after occupational exposure to HIV. Arch Intern Med 1997;157:1972-80. 